\title{
Level of Service Reliability for Stochastic Road Network Based on Travel Time Reliability
}

\author{
Ya Lei ${ }^{1, a}$, Aiwu Kuang ${ }^{1, b}$ and Gang Liu ${ }^{1, c}$ \\ ${ }^{1}$ School of Traffic and Transportation Engineering, Changsha University of Science and Technology, \\ Changsha 410004, P. R. China \\ a1198688298@qq.com, bjxgakaw@126.com, ‘857738752@qq.com
}

\begin{abstract}
Keywords: level of service reliability; travel time reliability; stochastic demand; travel time budget; traffic assignment

Abstract. To study the relationship of different reliability indices for road network under stochastic demand, an evaluation method based on travel time reliability (TTR) was set up for level of service (Los) reliability. Assuming that travelers acquire the variation of the route travel time based on past experiences and factor such variability into their route choice considerations in the form of travel time budget, which was defined as the minimal travel time that can accommodate a specified travel time reliability and can be derived according to the probability characteristics of log-normal distribution. Accordingly, Los reliability evaluation models of link and road network were proposed based on the link flow distribution, which can be determined by a travel time budget-based variational inequality (VI) network equilibrium model and a route-based solution algorithm. Some numerical studies on a small road network were presented to validate the proposed method and algorithm.
\end{abstract}

\section{Introduction}

Road network reliability is the ability of a road network to accomplish predetermined functions under specified conditions within a time interval. In recent years, with the development of economy and society, vehicles numbers and traffic demands has witnessed a sharp increase, while the traffic conditions have been worsened in China. Considerable work has been done to improve the reliability of stochastic road networks. From the viewpoint of system engineering, road network unreliability could be caused by uncertainties in both the supply and demand sides [1]. Many researches have been conducted on reliability analysis with stochastic supply and/or stochastic demand. These studies mainly focus on three aspects: connectivity, travel time reliability and capacity reliability. Although the existing studies about road network reliability have obtained a great of achievements, there still have some limitations worthy of further consideration. Firstly, the analysis on road network reliability lacks of a comprehensive evaluation index, few studies are available on the establishment of comprehensive evaluation indices from both the perspectives of road network user and system manager. Secondly, the studies on the relationship between different reliability indices are still lacking, such as one usually neglects the close association among connectivity, travel time and network capacity reliability when he/she carry out the road network reliability analysis. Thirdly, the formulations of the road network reliability evaluation models themselves are usually imperfect, for example, Lo et al. used uniform distribution to describe the random variation in link capacity, Shao et al. assumed that the traffic demand follows a normal distribution [2]. In fact, the determination of the probability distribution of traffic demand and link capacity is a key problem to road network reliability evaluation. Nevertheless, whether the distributions mentioned above can truly and properly reflect the stochastic variation in traffic supply and demand is still short of demonstration.

With the considerations that the level of service (Los) is an important index for individual user and system manager to measure the efficiency of a road network and to perceive the travel quality, which can be served as a comprehensive index for evaluating the road network performance. Therefore, in this paper, we develop a Los reliability evaluation method with the assumptions that the traffic demand follows a log-normal distribution and the traveler's route choice criterion is to minimize the route travel time budget. This paper is organized as follows. Section 2 is the model formulation. 
Section 3 presents a heuristic solution procedure. Section 4 is the numerical studies. Finally, section 5 concludes the paper and provides recommendations for future researches.

\section{Formulation}

\section{Route and link flow distributions}

Consider a road network represented by a directed graph $G(N, A)$, where $N$ is the set of nodes and $A$ is the set of links. Let $W$ be the set of OD pairs and $R_{w}$ be the set of routes between OD pair $w$. Denoted the demand of OD pair $w$ by $Q_{w}$, which is treated as a random variable and assumed to follow a log-normal distribution [3]. Let $q_{w}$ be the mean and $\varepsilon_{w}$ be the variance of OD pair $w$. Then,

$$
Q_{w} \sim \operatorname{Ln}\left(\mu_{\mathrm{q}, w}, \sigma_{\mathrm{q}, w}\right), \forall w .
$$

where $\mu_{\mathrm{q}, w}$ and $\sigma_{\mathrm{q}, w}$ are the mean and standard deviation of $\ln \left(Q_{w}\right)$, which can be computed by,

$$
\mu_{\mathrm{q}, w}=\ln \left(q_{w}\right)-0.5 \ln \left[1+\varepsilon_{w} /\left(q_{w}\right)^{2}\right], \sigma_{\mathrm{q}, w}=\left(\ln \left[1+\varepsilon_{w} /\left(q_{w}\right)^{2}\right]\right)^{0.5}, \forall w .
$$

Let $\sigma_{w}$ be the standard deviation of the traffic demand between OD pair $w$. Define the COV of demand as the ratio of the SD $\sigma_{w}$ to the mean $q_{w}$ as follows,

$$
\sigma_{w}=\sqrt{\varepsilon_{w}}, \operatorname{COV}_{w}=\sigma_{w} / q_{w}, \forall w
$$

Similar to the studies in Shao [1], we also assume that: (a) the route flow follows the same type of probability distribution as OD demand; (b) the route flow COV is equal to that of OD demand; (c) the route flows are mutually independent. Then, the flow conservation equations can be expressed as,

$$
Q_{w}=\sum_{k} F_{k w}, F_{k w} \geq 0, X_{a}=\sum_{w} \sum_{k} F_{k w} \delta_{a k w} .
$$

where $F_{k w}$ denotes the traffic flow on route $k$ between OD pair $w, X_{a}$ is the flow on link $a, \delta_{a k w}$ is the link-route incidence parameter whose value is one if link $a$ is on route $k$ and zero otherwise.

It is easy to see that $F_{k w}$ and $X_{a}$ are random variables. Let $f_{k w}$ and $x_{a}$ be the mean route flow and link flow, respectively. It follows from Eq. 4 that,

$$
q_{w}=\sum_{k} f_{k w}, f_{k w} \geq 0, x_{a}=\sum_{w} \sum_{k} f_{k w} \delta_{a k w} .
$$

According to the assumption (a) and (b), the route flows also follow log-normal distributions,

$$
F_{k w} \sim \operatorname{Ln}\left(\mu_{\mathrm{f}, k w}, \sigma_{\mathrm{f}, k w}\right), \mu_{\mathrm{f}, k w}=\ln \left(f_{k w}\right)-\frac{1}{2} \ln \left[1+\frac{\varepsilon_{\mathrm{f}, k w}}{\left(f_{k w}\right)^{2}}\right], \sigma_{\mathrm{f}, k w}=\left\{\ln \left[1+\frac{\varepsilon_{\mathrm{f}, k w}}{\left(f_{k w}\right)^{2}}\right]\right\}^{\frac{1}{2}}, \varepsilon_{\mathrm{f}, k w}=\left[\frac{\sigma_{w} f_{k w}}{q_{w}}\right]^{2} .
$$

where $\mu_{\mathrm{f}, k w}$ and $\sigma_{\mathrm{f}, k w}$ are the the mean and SD of $\ln \left(F_{k w}\right), \varepsilon_{\mathrm{f}, k w}$ is the variance of the route flow.

Based on the route-link relationship in Eq. 4, the log-normal distribution parameters $\mu_{\mathrm{x}, a}$ and $\sigma_{\mathrm{x}, a}$ of the random link flow can be approximated as follows [4].

$$
X_{a} \sim \operatorname{Ln}\left(\mu_{\mathrm{x}, a}, \sigma_{\mathrm{x}, a}\right), \mu_{\mathrm{x}, a}=\ln \left(x_{a}\right)-\frac{1}{2} \ln \left[1+\frac{\varepsilon_{\mathrm{x}, a}}{\left(x_{a}\right)^{2}}\right], \sigma_{\mathrm{x}, a}=\left\{\ln \left[1+\frac{\varepsilon_{\mathrm{x}, a}}{\left(x_{a}\right)^{2}}\right]\right\}^{\frac{1}{2}}, \varepsilon_{\mathrm{x}, a}=\sum_{w} \sum_{k}\left[\frac{\sigma_{w} f_{k w}}{q_{w}}\right]^{2} \delta_{a k w} .
$$




\section{Link and route travel time distributions}

In this paper, we adopt the BPR link performance function, which defines the link travel time, $T_{a}$, under flow $X_{a}$ using two deterministic parameters $\alpha$ and $\beta$ as,

$$
T_{a}\left(X_{a}\right)=t_{a 0}\left(1+\alpha\left(X_{a} / c_{a}\right)^{\beta}\right) \text {. }
$$

where $t_{a 0}, c_{a}$ are the deterministic free-flow travel time and capacity on link $a$, respectively.

Using the probability characteristics of log-normal link flow, the mean and variance of the link travel time based on the BPR function can be derived as follows,

$$
t_{a}=t_{a 0}+\frac{\alpha t_{a 0}}{\left(c_{a}\right)^{\beta}} e^{\beta \mu_{\mathrm{x}, a}+\frac{1}{2} \beta^{2}\left(\sigma_{\mathrm{x}, a}\right)^{2}}, \varepsilon_{\mathrm{t}, a}=\left(\frac{\alpha t_{a 0}}{\left(c_{a}\right)^{\beta}}\right)^{2} \times\left[e^{2 \beta \mu_{\mathrm{x}, a}+\beta^{2}\left(\sigma_{\mathrm{x}, a}\right)^{2}}\right] \times\left[e^{\beta^{2}\left(\sigma_{\mathrm{x}, a}\right)^{2}}-1\right] .
$$

where $t_{a}, \varepsilon_{\mathrm{t}, a}$ are the mean and variance of link travel time $T_{a}$, respectively.

To facilitate the presentation of the essential ideas, this paper assumes that the link travel times are independent. Hence, using the route-link incidence relationship, the random travel time $T_{k w}$ on route $k$ between OD pair $w$ can be simply expressed as the summation of the independent link travel time variables along route $k$. According to the Central Limit Theorem, in a network with routes consisting of many links, the route travel time follows a normal distribution regardless of the link travel time distributions. Therefore, the mean route travel time $t_{k w}$, and the route travel time standard deviation $\sigma_{\mathrm{t}, k w}$ can be expressed as,

$$
\left.t_{k w}=\sum_{a} \delta_{a k w}\left\{t_{a 0}+\frac{\alpha t_{a 0}}{\left(c_{a}\right)^{\beta}} e^{\beta \mu_{x, a}+\frac{1}{2} \beta^{2}\left(\sigma_{x, a}\right)^{2}}\right\}, \sigma_{\mathrm{t}, k w}=\sqrt{\sum_{a} \delta_{a k w}\left\{\frac{\left(\alpha t_{a 0}\right)^{2}}{\left(c_{a}\right)^{2 \beta}}\left[e^{2 \beta \mu_{x, a}+\beta^{2}\left(\sigma_{x, a}\right)^{2}}\right] \times\left[e^{\beta^{2}\left(\sigma_{x, a}\right)^{2}}-1\right]\right.}\right\} .
$$

\section{Model of route travel time budget}

In this paper, the route travel time budget $b_{k w}$ is defined as the summation of the mean route travel time and the safety margin $m_{k w}$ and can be expressed as: $b_{k w}=t_{k w}+m_{k w}, \forall w \in W, k \in R_{w}$. Mathematically, route travel time budget can be determined by the following chance-constrained model,

$\min b_{k w}$.

s.t. $\operatorname{Pr}\left[T_{k w} \leq b_{k w}\right] \geq \rho \forall w \in W, k \in R_{w}$.

where $\rho$ is the travel time reliability requirement. Since $T_{k w}$ follows a normal distribution, $b_{k w}$ can be obtained by directly solving constraint (12). As a result, $m_{k}{ }^{w}$ and $b_{k}{ }^{w}$ can be expressed as,

$$
m_{k w}=\sigma_{\mathrm{t}, k w} \Phi^{-1}(\rho) \quad b_{k w}=t_{k w}+\sigma_{\mathrm{t}, k w} \Phi^{-1}(\rho) \quad \forall w \in W, k \in R_{w}
$$

where $\Phi(x)$ is the standard normal cumulative function of random variable $x$.

\section{Route choice behavior and VI formulation}

In this paper, it is assumed that the deterministic user equilibrium (UE) is reachable under stochastic demands with a log-normal distribution. At the equilibrium state, for each OD pair, no travelers can improve their route travel time budget by unilaterally changing routes. In other words, all used routes between each OD pair have equal travel time budget, and no unused route has a lower

travel time budget. Let vector $\mathbf{b}$ be the route travel time budget $\left(\ldots, b_{k w}, \ldots\right)^{\mathrm{T}}, \pi_{w}$ denotes the minimal 
travel time budget between OD pair $w$, f denotes the route flow vector $\left(\ldots, f_{k w}, \ldots\right)^{\mathrm{T}}$. Then, the UE conditions can be expressed as,

$$
b_{k w}-\pi_{w}\left\{\begin{array}{lll}
=0 & \text { if } \quad f_{k w}>0 \\
\geq 0 & \text { if } \quad f_{k w}=0
\end{array} .\right.
$$

Mathematically, the UE conditions can be formulated as an equivalent VI problem as follows.

Find a mean route flow vector $\mathbf{f}^{*} \in \Omega$, such that:

$$
\mathbf{b}^{\mathrm{T}}\left(\mathbf{f}^{*}\right)\left(\mathbf{f}-\mathbf{f}^{*}\right) \geq 0, \forall \mathbf{f} \in \Omega
$$

where the superscript "**" is used to designate the solution of the VI problem; $\Omega$ represents the feasible set for the mean route flow vector defined by Eq. 5 .

\section{Los reliability evaluation model}

Once the distribution of link flow is determined, one can carry out the Los reliability evaluation with uncertainties in traffic demands. To facilitate the calculation, in this paper, the degree of congestion is used as a Los measurement index. Following the traffic survey, the common classification criteria on the degree of congestion for different Los levels are given in Table 1 [5].

Table 1 Urban road Los division value

\begin{tabular}{|c|c|c|c|c|}
\hline Los & Level 1 & Level 2 & Level 3 & Level 4 \\
\hline Degree of congestion & $<0.55$ & $0.55-0.75$ & $0.75-0.9$ & $>0.9$ \\
\hline
\end{tabular}

Let $\theta_{1, i}, \theta_{2, i}$ be the degree of congestion representing the best and the worst service quality corresponding to $i$ th level of service, respectively. Through the link flow distribution, the link Los reliability evaluation model can be expressed as,

$$
R_{a}^{i}=P_{r}\left[\theta_{1, i} \leq \frac{X_{a}}{c_{a}} \leq \theta_{2, i}\right]=\Phi\left[\frac{\ln \left(\theta_{2, i} c_{a}\right)-\mu_{\mathrm{x}, a}}{\sigma_{\mathrm{x}, a}}\right]-\Phi\left[\frac{\ln \left(\theta_{1, i} c_{a}\right)-\mu_{\mathrm{x}, a}}{\sigma_{\mathrm{x}, a}}\right] .
$$

where $R_{a}{ }^{i}$ denotes the Los reliability of link $a$ maintaining at the $i$ th Los.

In this paper, the degree of congestion of a road network is defined as the weighted average of the degree of congestion of each link in the network.

$$
\delta_{G}=\sum_{a} l_{a} X_{a} / \sum_{a} l_{a} c_{a}
$$

where $\delta_{G}$ denotes the degree of congestion of a road network, $l_{a}$ is the length of link $a$.

Similar to the evaluation of link Los reliability, the model for evaluating road network Los reliability is as shown below,

$$
R_{G}^{i}=\Phi\left[\frac{\ln \left(\theta_{2, i} \sum_{a} l_{a} c_{a}\right)-\mu_{G}}{\sigma_{G}}\right]-\Phi\left[\frac{\ln \left(\theta_{1, i} \sum_{a} l_{a} c_{a}\right)-\mu_{G}}{\sigma_{G}}\right] .
$$

where $R_{G}{ }^{i}$ denotes the Los reliability of the road network maintaining at the $i$ th Los. $\mu_{G}, \sigma_{G}$ denote the parameters of the log-normal distribution of network flow, which can be calculated using the following formulae. 


$$
\mu_{G}=\ln \left(\sum_{a} l_{a} x_{a}\right)-\frac{1}{2} \ln \left[1+\frac{\sum_{a}\left(l_{a}\right)^{2} \varepsilon_{\mathrm{x}, a}}{\left(\sum_{a} l_{a} x_{a}\right)^{2}}\right], \sigma_{G}=\left\{\ln \left[1+\frac{\sum_{a}\left(l_{a}\right)^{2} \varepsilon_{\mathrm{x}, a}}{\left(\sum_{a} l_{a} x_{a}\right)^{2}}\right]\right\}^{\frac{1}{2}} .
$$

\section{Solution algorithm}

In this section, a solution algorithm is developed. It should be noted that a number of well-known algorithms developed for traditional traffic assignment problems, such as Frank-Wolfe algorithm cannot be directly applied to solve model (15) due to the non-additive property of route travel time budget. Under this circumstance, a route-based algorithm is available for solving the proposed VI model [6]. The step-by-step procedure of this algorithm is given below.

Step 0: Initialization. Define the stopping tolerance $\tau$, set iteration counter $n=0$, determine the assignment route set with column generation algorithm, generate an initial route flow pattern $\mathbf{f}^{(0)}$.

Step 1: Update. Update the route travel time budget vector $\mathbf{b}^{(n)}$ based on the current route flow $\mathbf{f}^{(n)}$.

Step 2: Direction finding. Perform the all or nothing assignment in terms of the updated route travel time budget $\mathbf{b}^{(n)}$ and get the auxiliary route flow $\mathbf{y}^{(n)}$.

Step 3: Move. Find a new route flow pattern based on the method of successive averages (MSA), namely, $\mathbf{f}^{(n+1)}=\mathbf{f}^{(n)}+\left(\mathbf{y}^{(n)}-\mathbf{f}^{(n)}\right) /(n+1)$.

Step 4: Convergence check. If a certain equilibrium criterion is reached, then stop and report the solution; otherwise, set $n=n+1$ and go to Step 1 .

\section{Numerical studies}

A simple example road network given in Fig. 1 consists of four nodes, five links, and one OD pair (from node 1 to node 4). It is assumed that the demand of OD pair $Q_{14}$ follows log-normal distribution with mean demand $q_{14}=800(\mathrm{pcu} / \mathrm{h})$. The link travel time function adopted in this study is the BPR function (Eq. 8) with parameters $\alpha=0.15$ and $\beta=2$. The convergence precision used in the traffic assignment model is $\tau=0.01$. Other parameters, including the length of each link, link free-flow travel time and link capacity are as shown in Table 2.

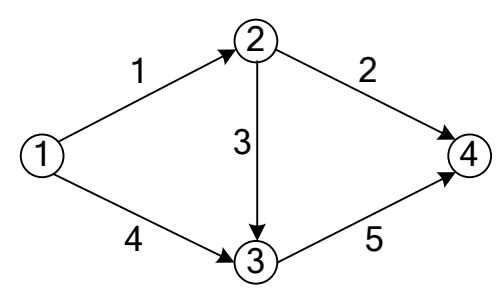

Fig. 1 Test network

Table 2 The length of link, link free flow travel time and link capacity

\begin{tabular}{|c|c|c|c|}
\hline Link no. & Length $(\mathrm{km})$ & Free flow travel time $(\mathrm{min})$ & Capacity $(\mathrm{pcu} / \mathrm{h})$ \\
\hline 1 & 2 & 5 & 600 \\
\hline 2 & 2 & 13 & 400 \\
\hline 3 & 2 & 4 & 400 \\
\hline 4 & 2 & 8 & 400 \\
\hline 5 & 2 & 10 & 600 \\
\hline
\end{tabular}


According to the calculation results, at the equilibrium state, the traffic flows on link 1-5 are 508, 303, 205, 292, 497 ( $\mathrm{pcu} / \mathrm{h}$ ), respectively, and the equilibrium travel time budget is 19.783 (min) for three routes connecting node 1 to node 4. However, as can be seen in Fig. 2, the Los reliabilities of the five links in equilibrium state are different from one another. The Los reliability is mainly maintained at Level 3 for Link 1 and Link 5, Level 1 for Link 3, and Level 2 or Level 3 for Link 2 and Link 4 and the whole network. The results show that under the action of stochastic demand, the Los reliabilities of link and network are no longer a fixed value, rather, they will vary with the stochastic demand.

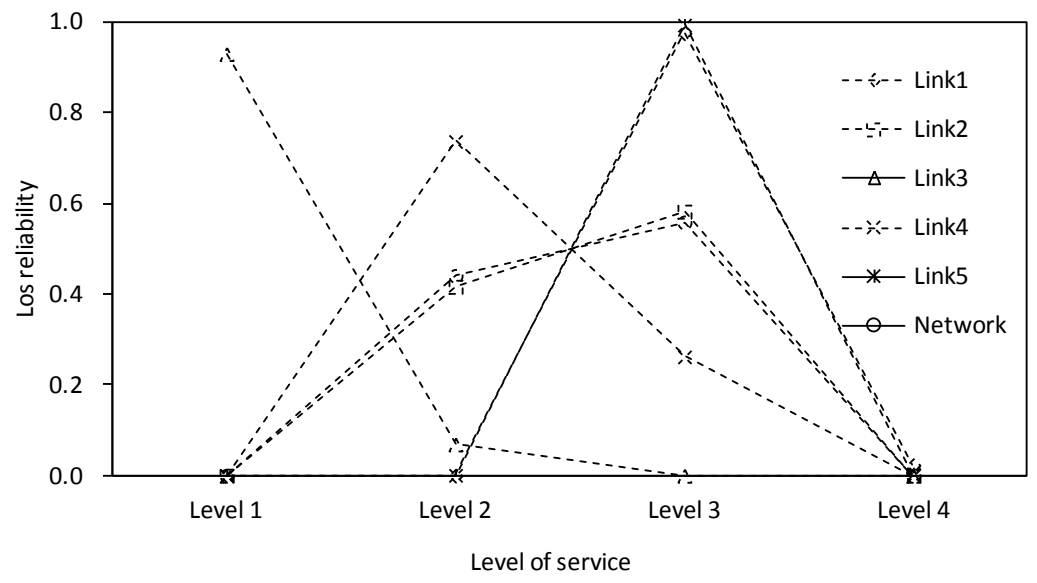

Fig. 2 Los reliability evaluation results of each link

Consider the impact of traffic demand levels on Los reliability, the basic demand of OD pair $(1,4)$ is set to be $q_{14}=250(\mathrm{pcu} / \mathrm{h})$ and the $\mathrm{COV}_{14}$ equals to 1 , the travel time reliability requirement is specified to be $\rho=0.9$. Mean traffic demand of different demand levels is measured by $q=\lambda \cdot q_{14}$, where $\lambda=(1.0,1.2, \ldots, 4.8,5.0)$ is the demand multiplier that represents the demand level from 1 to 21, respectively. Fig. 3 depicts the Los reliability of the road network maintaining at various Los with respect to different demand levels. From the figure, we can find that a higher demand will lead to a gradually decrease of Los reliability if the service level is specified at a high level, and vice versa. Nevertheless, when the Los is specified at level 2 or level 3, we can observe that the Los reliability moves upwards first and then downwards. These results reveal that the traffic demand level has significant impact on Los reliability, once the demand level exceeds a critical value, the Los reliability drops rapidly.

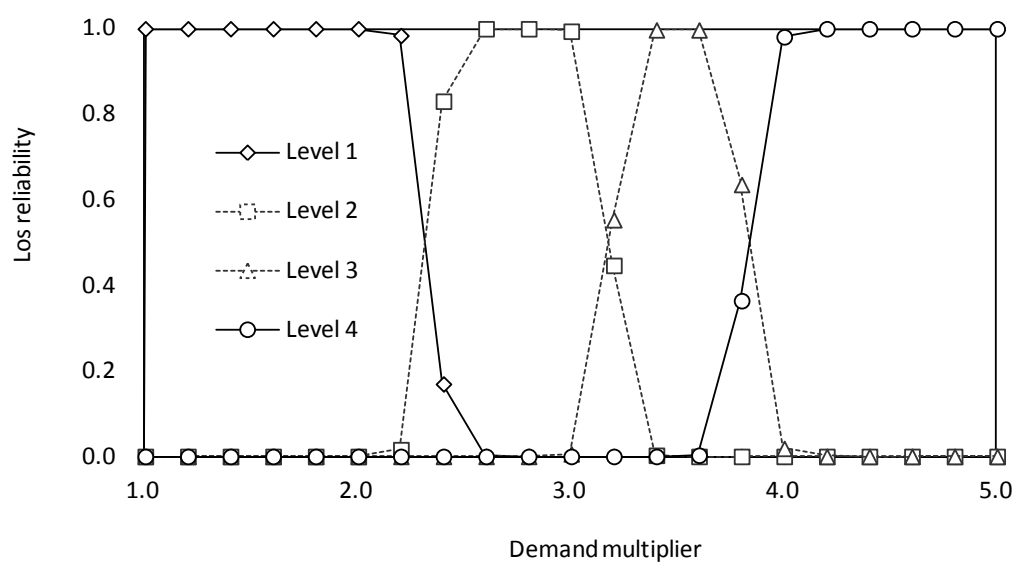

Fig. 3 Los reliability with different OD demand multiplier 


\section{Conclusions}

The impact of random demand on road network reliability is investigated and a travel time reliability based method for evaluating the Los reliability of link and road network under stochastic demand is proposed in this paper. Some numerical analyses of applying the proposed evaluation method on a small test network have been carried out to demonstrate its applicability and efficiency. The numerical studies show that: 1) the randomness of traffic demand will lead to the random changes in the level of service and influence the Los reliability of link and road network with a travel time reliability constraint; 2) the traffic demand level has significant impact on the Los reliability and reducing traffic demand by avoiding unnecessary traffic is important to the improvement of network efficiency and reliability.

\section{Acknowledgements}

This research was supported in part by the National Natural Science Foundation of China under grant number 51208064, the Science and Technology Plan Project of Hunan Province under research grant 2015SK2012, and the Open Fund of MOE Engineering Research Center of Catastrophic Prophylaxis and Treatment of Road \& Traffic Safety (Changsha University of Science \& Technology) under research grant kfj130301.

\section{REFERENCES}

[1] Shao, H., Lam, W. H. K., Tam, M. L., 2006. A reliability-based stochastic traffic assignment model for network with multiple user classes under uncertainty in demand. Network and Spatial Economics, 6(3): 173-204.

[2] Lo, H. K., Luo, X. W., Siu, B. W. Y., 2006. Degradable transport network: Travel time budget of travelers with heterogeneous risk aversion. Transportation Research Part B, 40(9): 792-806.

[3] Zhou, Z., Chen, A., 2008. Comparative analysis of three user equilibrium models under stochastic demand. Journal of Advanced Transportation, 42(3): 239-263.

[4] Fenton, L. F., 1960. The sum of log-normal probability distributions in scatter transmission systems. IEEE Transactions on Communications Systems, 8(1): 57-67.

[5] Wang, W., Xu, J. Q., Yang, T., Li, X. H., 1998. Urban traffic planning theory and its application. Southeast University Press, Nanjing.

[6] Lam, W. H. K., Chan, K. S., Li, Z. C., 2010. A risk-averse user equilibrium model for route choice problem in signal-controlled networks. Journal of Advanced Transportation, 44(4): 219-230. 